\title{
Considerazioni medico-legali sulla mozione del Comitato Nazionale per la Bioetica sull'accanimento clinico nei confronti di bambini con limitate aspettative di vita
}

\author{
Anna Aprile, Matteo Bolcato, Marianna Russo, Daniele Rodriguez \\ Medicina Legale, Dipartimento di Medicina Molecolare, Università degli Studi di Padova
}

La mozione del Comitato Nazionale per la Bioetica (CNB) italiano del 30 gennaio 2020, intitolata "Accanimento clinico o ostinazione irragionevole dei trattamenti sui bambini piccoli con limitate aspettative di vita”, enuncia 12 raccomandazioni, di carattere eminentemente pratico, rivolte sostanzialmente ai medici curanti che hanno in cura questi bambini e che devono confrontarsi con i genitori per fornire le informazioni necessarie e per sostenerli nel processo di valutazione e nella decisione conclusiva. Ciascuna raccomandazione è analiticamente commentata.

La raccomandazione 1 attribuisce un ruolo centrale alla pianificazione condivisa delle cure gestita dall'intera équipe medica, sostiene il trattamento del dolore e il sollievo della sofferenza, contrasta i trattamenti clinici inefficaci e sproporzionati, osteggia la medicina difensiva. La raccomandazione 2 auspica una legge nazionale che istituisca e renda operativi i comitati per l'etica clinica negli ospedali pediatrici. La raccomandazione 3 propone la partecipazione dei genitori e di loro fiduciari ai processi decisionali dei medici e dei comitati per l'etica clinica. La raccomandazione 4 enuncia la possibilità del ricorso a una seconda opinione che può essere richiesta sia dei genitori sia dell'équipe curante. La raccomandazione 5 ammette, come extrema ratio, il ricorso ai giudici in caso di insanabile disaccordo fra l'équipe medica e i familiari. La raccomandazione 6 proclama l'accompagnamento del morire da realizzare anche attraverso la sedazione profonda continua in associazione con la terapia del dolore. La raccomandazione 7 enuncia il principio di garantire l'accesso alle cure palliative. La raccomandazione 8 ribadisce la necessità di potenziare la ricerca sul dolore e sulla sofferenza nei bambini. La raccomandazione 9 esclude che la sopravvivenza possa essere legata meramente al portare a termine un progetto di sperimentazione e ricerca. La raccomandazione 10 riguarda la formazione dei medici, del personale sanitario e degli psicologi, per sostenere i genitori sul piano emotivo e pratico. La raccomandazione 11 esorta ad agevolare la vicinanza dei genitori ai bambini in condizioni cliniche estremamente precarie. La raccomandazione 12 conferisce un ruolo di rilievo delle associazioni dei genitori di bambini malati.

The motion approved by the Italian National Bioethics Committee January 30", 2020, entitled "Aggressive treatments or unreasonable obstinacy in medical care of children with short life expectancy", sets out 12 recommendations for physicians who take care of these children and have the duty to communicate with their parents. The intent of this motion is to give the family the necessary information and support during the decision-making process. Each recommendation is analytically commented.

Recommendation 1 advocates the adoption of shared decision-making by the entire healthcare team; promotes the relief of pain and suffering; inhibits disproportionate and ineffective medical treatments; opposes a defensive medicine. Recommendation 2 asks for the promulgation of a national law that establishes clinical ethics committees in Children's Hospitals. Recommendation 3 implies that parents or legal guardians should be involved in the decision-making process of physicians and clinical ethics committees. Recommendation 4 states that a second opinion may be requested by both the parents and the physicians. Recommendation 5 admits an appeal to the judge in the event of an irreconcilable conflict between the health care team and the family. Recommendation 6 endorses the medical accompaniment of the dying patient, also by means of continuous deep sedation and analgesia. Recommendation 7 enunciates the principle that patients shall have the right to access to palliative care. Recommendation 8 confirms the necessity to strengthen research focused on pediatric pain and suffering. Recommendation 9 excludes that excessive medical treatment may be justified by the need to carry out experiments or research projects. Recommendation 10 concerns the training of physician, other healthcare workers and psychologists in order to support children's parents both emotionally and practically. Recommendation 11 exhorts to encourage the interaction between parents and seriously ill children. Recommendation 12 assigns a prominent role to parents' associations for ill children.

\section{Introduzione}

Quando ci si interroga sulle terapie da praticare a un bambino piccolo, occorre essere rispettosi della sua dignità e individuare quale sia il suo migliore interesse, considerando le caratteristiche della patologia, le potenzialità e i limiti delle cure possibili nonché i costi, in senso lato, e i benefici attesi. Ogni atto medico deve tener con- to dei criteri di appropriatezza e proporzionalità; interventi eseguiti esclusivamente al fine di prolungare la vita fisica e che non considerino l'interesse generale della persona non sono rispettosi della dignità della stessa. Nell'adulto, la proporzionalità tra i costi e i benefici tiene conto anche del giudizio dell'interessato, che la interpreta e la misura alla luce dei propri valori e della propria visione della vita. Nel caso del bambino, di norma, questi aspetti sono valutati dai suoi genitori, in quanto tutori naturali dello stesso [1].

Quando si tratta di bambini piccoli con prognosi sicuramente infausta e aspettativa di vita limitata e per i quali si prospettano trattamenti inefficaci o inappropriati, spesso gravosi, la valutazione dei genito- 


\section{BOX 1. Le 12 raccomandazioni contenute nella mozione del CNB del 30 gennaio 2020, intitolata "Accanimento} clinico o ostinazione irragionevole dei trattamenti sui bambini piccoli con limitate aspettative di vita".

1. Identificare l'accanimento clinico attraverso dati scientifici e clinici il più possibile oggettivi, garantendo la migliore qualità dei trattamenti disponibili e avvalendosi possibilmente di una pianificazione condivisa tra l'équipe medica e i genitori nel superiore interesse del bambino. II superiore interesse del bambino è il criterio ispiratore nella situazione e deve essere definito a partire dalla condizione clinica contingente, unitamente alla considerazione del dolore e della sofferenza (per quanto sia possibile misurarli), e del rispetto della sua dignità, escludendo ogni valutazione in termini di costi economici. Si deve evitare che i medici si immettano in percorsi clinici inefficaci e sproporzionati solo per accondiscendere alle richieste dei genitori e/o per rispondere a criteri di medicina difensiva.

2. Istituire per legge nazionale e rendere effettivamente operativi i comitati per l'etica clinica negli ospedali pediatrici con ruolo consultivo e formativo, così da favorire la valutazione della complessità di tali decisioni e cercare una mediazione di controversie emergenti tra medici e genitori. Tali comitati etici dovranno essere interdisciplinari, composti da medici pediatri, specialisti degli ambiti medici oggetto di analisi, infermieri, e figure non sanitarie quali bioeticisti e biogiuristi. I comitati non dovranno sostituirsi ai professionisti nell'adottare le decisioni del caso, ma aiutarli a esercitare in modo responsabile la propria autonomia.

3. Integrare i processi decisionali dei medici e dei comitati etici, con la partecipazione dei genitori e di persone di loro fiducia, garantendo lo spazio e il tempo della comunicazione e della riflessione, coinvolgendoli nel piano di cura e di assistenza del piccolo paziente, informandoli adeguatamente sul possibile evolversi della patologia in atto al fine di identificare i limiti degli interventi terapeutici e la legittimità di iniziare i trattamenti di cura o di mantenerli o di sospenderli facendo ricorso alle cure palliative.

4. Consentire una eventuale seconda opinione, rispetto a quella dell'équipe che per prima ha preso in carico il bambino, se richiesta dai genitori o dall'équipe curante, garantendo, in condizione di autorevolezza scientifica, la libertà di scelta dei genitori, tenuto conto del primario interesse del figlio. L'auspicio del CNB è che le due opinioni possano dare maggiore certezza della identificazione dell'accanimento clinico e una maggiore condivisione nell'iniziare 0 continuare o sospendere i trattamenti in corso. A tal fine è opportuno rendere facilmente accessibile l'intera documentazione clinica del paziente (cartella clinica e fascicolo sanitario elettronico), così da potere valutare il giudizio scientifico ed etico in merito al ritenuto accanimento clinico.

5. Prevedere il ricorso ai giudici, in caso di insanabile disaccordo tra l'équipe medica e i familiari, come extrema ratio e nel rispetto della legge 219/2017; tale soluzione andrebbe presa in considerazione solo dopo avere cercato una mediazione attraverso un'adeguata comunicazione con i genitori o la famiglia, tenendo conto di una corretta documentazione clinica e della richiesta al comitato di etica clinica.

6. Evitare che il divieto di ostinazione irragionevole dei trattamenti si traduca nell'abbandono del bambino nei cui confronti da parte dei medici resta fermo l'assoluto dovere di trattamenti e sostegni appropriati, siano essi presidi tecnologici o farmacologici, e di cure palliative con l'accompagnamento nel morire, anche attraverso la sedazione profonda continua in associazione con la terapia del dolore.

7. Garantire l'accesso alle cure palliative, ospedaliere e a domicilio, in modo omogeneo sul territorio.

8. Potenziare la ricerca sul dolore e sulla sofferenza nei bambini, al fine di implementare e migliorare la validazione di scale di misurazione oggettive del dolore e della sofferenza, che possano orientare - insieme ad altri parametri - le decisioni cliniche.

9. Evitare che il bambino, a maggior ragione con prognosi infausta a breve termine, sia considerato un mero oggetto di sperimentazione e ricerca da parte dei medici.

10. Implementare la formazione dei medici, del personale sanitario e degli psicologi, per creare un nucleo di professionisti in grado di sostenere i genitori sul piano emotivo e pratico (assistenti sociali, psicologi, esperti di bioetica, associazioni delle famiglie, associazioni di volontariato) e accompagnarli nel difficile percorso, dato dalle condizioni di malattia del bambino.

11. Agevolare la vicinanza dei genitori ai bambini in condizioni cliniche estremamente precarie (es. cure palliative svolte nella propria abitazione; previsione di congedi dal lavoro, ecc.).

12. Riconoscere il ruolo di rilievo delle associazioni dei genitori di bambini malati e consolidare le reti per l'aiuto solidale ai genitori anche da parte della società.

ri diviene particolarmente complessa ed è indispensabile che sia basata su elementi clinici analitici e su un definito progetto di cura [2], chiaramente illustrati loro dall'équipe curante. Questa dovrà altresì accompagnare i genitori nel difficile processo di accettazione della prognosi infausta della malattia e della inappropriatezza o dell'inefficacia di determinati trattamenti intensivi/invasivi, fugando l'eventuale preconcetto che astenersi da tali trattamenti equivalga a sospendere il percorso di cura, che invece è garantito attraverso una terapia palliativa $[3,4]$.

Non raramente, il confronto fra équipe curante e genitori [5] fa emergere criticità legate anche ad alcuni concetti fondamentali per affrontare le questioni appena indicate. La stessa nozione di interesse [6], per esempio, non è di facile definizione, soprattutto quanto più il bambino è piccolo. Gli elementi caratterizzanti l'interesse possono rientrare in due categorie principali: quelli di natura biologica (integrità, funzionalità, sofferenza, dolore, coscienza) e quelli di natura antropologico-affettivo-relazionale (essere oggetto e soggetto di affetto per più persone oltre ai genitori, relazioni con altri, desideri, esperienze, espressione di sé, apprendimento, gestione dei cambiamenti e altro ancora). I primi sono individuati dal medico, i secondi sono percepiti dai genitori. L'uno e gli altri (questi ultimi talora non concordi fra loro) non hanno, necessariamente, la stessa idea di interesse del bambino.

Occorre pertanto che l'interesse del bambino sia considerato anche rispetto agli elementi antropologico-affettivo-relazionali evidenziati dai genitori [7]. Nella pra- tica, può capitare che essi maturino un'opinione favorevole a trattamenti intensivi/ invasivi ritenendo sussistente, in base a questi ultimi criteri, l'interesse del bambino e quindi il requisito dell'appropriatez$z a$, negati invece dai medici in base ai criteri di natura biologica. In caso di difformità di valutazione degli interessi e quindi dell'appropriatezza, è da considerare che solo i genitori hanno percezione degli elementi antropologico-affettivo-relazionali in funzione del loro rapporto di condivisione di esperienza di vita col bambino e pertanto è da ritenere prevalente la valutazione dei genitori, a condizione che la loro opinione sia espressa dopo colloqui approfonditi, svolti con calma, tesi a consentire loro di esprimere dubbi, paure e desideri e di procedere a valutazioni consapevoli, pur nella situazione di grande dolore e stress 
in cui si trovano [8]. È possibile che questi colloqui inducano il medico a modificare la propria iniziale valutazione dell'interesse del bambino e ad accogliere le opinioni dei genitori [9].

Il giudizio di inefficacia di un determinato trattamento, fondato necessariamente su basi scientifiche, è di pertinenza medica, ma anch'esso dovrà essere ben illustrato ai genitori, sempre tenendo presente la loro difficile situazione ed eventualmente suggerendo loro di ricorrere a un consulto. L'inefficacia del trattamento, per quanto richiesto dai genitori, impone al medico di astenersi dal praticarlo.

Si tratta di valutazioni molto complesse, che comportano decisioni assai dolorose per i genitori, che devono riuscire a sceverare il piano emotivo da quello razionale. Il medico deve essere in grado di instaurare con i genitori un rapporto chiaro, nel contesto del quale fornire adeguate motivazioni di un suo eventuale giudizio di inappropriatezza o di inefficacia di un dato trattamento e saper ascoltare le considerazioni dei genitori circa la dignità da riconoscere al piccolo bambino.

La mozione del Comitato Nazionale per la Bioetica (CNB) del 30 gennaio 2020, intitolata "Accanimento clinico o ostinazione irragionevole dei trattamenti sui bambini piccoli con limitate aspettative di vita", dopo un'analitica premessa, enuncia 12 raccomandazioni, rivolte ai medici che hanno in cura questi bambini e che devono confrontarsi con i genitori per fornire le informazioni necessarie e per sostenerli nel processo di valutazione e nella decisione conclusiva (Box 1).

Nell'ultima parte di detta premessa, si considera che il tema "sebbene appaia già consolidato in bioetica, è in verità ancora molto incerto (consapevolmente o inconsapevolmente) nella prassi”. Le 12 raccomandazioni hanno dunque significato pratico. Pertanto, svolgiamo un'analisi delle 12 raccomandazioni tenendo conto degli aspetti concreti che, in base anche alla nostra lunga esperienza, sia di consulenze ospedaliere di bioetica clinica [10] sia di partecipazione a un comitato etico per la pratica clinica pediatrica, più spesso assumono importanza nelle singole vicende.

Analisi delle 12 raccomandazioni del CNB Analizziamo le 12 raccomandazioni, limitandoci a discutere gli aspetti di non chiara interpretazione e quelli innovativi o comportanti problemi nella applicazione pratica.

\section{Raccomandazione 1}

La raccomandazione 1 attribuisce un ruolo centrale alla pianificazione condivisa. Si tratta dell'applicazione all'ambito di specie della previsione dell'art. 5 della legge 22 dicembre 2017, n. 219 [11,12]. La raccomandazione 1 non specifica che si tratta di pianificazione "delle cure" come fa l'art. 5. E comunque evidente che la pianificazione condivisa ha quale obiettivo la scelta delle modalità più appropriate di "prendersi cura” del piccolo paziente.

La pianificazione condivisa delle cure è attuabile in ogni struttura sanitaria, che, in forza del comma 9 dell'art. 1 della legge predetta, "garantisce con proprie modalità organizzative la piena e corretta attuazione dei principi di cui alla presente legge, assicurando l'informazione necessaria ai pazienti e l'adeguata formazione del personale". Saggiamente, la raccomandazione 1 non limita a un medico la partecipazione alla pianificazione, come previsto dall'art. 5 della legge 219, ma la estende all'intera équipe medica, da intendere, più estensivamente, come équipe sanitaria, includente cioè tutti i professionisti curanti. La pianificazione va promossa nell'interesse del minore e l'unica ragionevole "possibilità" (il richiamo è all'avverbio "possibilmente" usato nella raccomandazione) di non attuarla è data dal rifiuto dei genitori, che devono comunque essere adeguatamente informati su questo strumento. La pianificazione condivisa delle cure agevola l'ascolto e la comunicazione reciproci ed è mezzo ideale per affrontare nel concreto le caratteristiche della malattia, la sua prevedibile evoluzione, i trattamenti possibili, le aspettative dei genitori.

$\mathrm{La}$ "considerazione del dolore e della sofferenza", da valorizzare in rapporto al superiore interesse del bambino, è completata da un'indicazione riportata fra parentesi: "per quanto sia possibile misurarli". Il termine "possibile" va qui inteso non in senso riduttivo come facoltà, ma quale esortazione ad adottare tutti gli strumenti disponibili per rilevare dolore e sofferenza del bambino $[13,14]$. In argomento, ricordiamo l'art. 3 del codice di deontologia medica, che cita fra i doveri del medico "il trattamento del dolore e il sollievo della sofferenza", le linee guida per la realizzazione dell'"ospedale senza dolore" allegate al provvedimento della conferenza permanente per i rapporti tra lo Stato, le Regioni e le Province Autonome di Trento e Bolzano 24 maggio 2001 nonché la legge 15 marzo 2010 n. 38 "Disposizioni per garantire l'accesso alle cure palliative e alla terapia del dolore".

Le richieste dei genitori sono viste come potenzialmente suggestive nei confronti del pediatra curante e tali da poter indurre "percorsi clinici inefficaci e sproporzionati”. In realtà le richieste dei genitori sono meritevoli di ascolto e di attenzione, come espressione dei loro bisogni e dei loro desideri. Come tali vanno dunque prese in considerazione e rapportate ai dati clinici e alle concrete possibilità di accoglierle nell'interesse del bambino.

La inammissibilità di trattamenti somministrati per ragioni di medicina difensiva allude al condizionamento che può essere indotto dall'atteggiamento dei genitori o dall'idea preconcetta del medico che immagini che i genitori interpretino come non appropriata la scelta di non attuare determinati trattamenti da loro richiesti e scelgano pertanto di rivendicare le loro ragioni in sede giudiziaria. La sistematica adozione dello strumento della pianificazione condivisa delle cure è idonea a fare chiarezza fra aspettative dei genitori e potenzialità scientifiche e limitare condizionamenti dovuti ad atteggiamenti di genitori, che non sono stati resi consapevoli delle caratteristiche della malattia e delle risorse della scienza medica.

\section{Raccomandazione 2}

La raccomandazione 2 auspica una legge nazionale che istituisca e renda operativi i comitati per l'etica clinica negli ospedali pediatrici; puntualmente ne identifica i ruoli consultivo e formativo [15], da intendere rivolti sia ai professionisti sanitari sia ai genitori. È corrispondente ai bisogni l'esistenza di un comitato per l'etica clinica specificamente competente in relazione all'area pediatrica. Il richiamo alle "controversie" può far intendere che il ricorso al comitato etico sia limitato ai casi in cui si sia instaurato un rapporto conflittuale fra équipe curante e genitori; in realtà, il ruolo consultivo del comitato etico è quello di "favorire la valutazione della complessità di tali decisioni” a prescindere da eventuali controversie. Lascia perplessi l'espressione che rinvia a un'attività di "mediazione" del comitato etico, perché un siffatto intervento, volto a comporre l'ipotizzata controversia, contrasta con il ruolo consultivo in precedenza correttamente dichiarato. Concordiamo con il fatto che i citati comitati etici siano interdisciplinari ma rilievi sono da fare al proposto elenco dei suoi componenti: non figurano professionisti sanitari spesso coinvolti nella cura del bambino terminale, quali logopedisti, fisioterapisti e dietisti; sono citati gli infermieri, ma non gli infermieri pediatrici, figura espressamente creata, con D.M. 17 gennaio 1997 n. 70, per l'assistenza dei bambini; mancano, fra gli altri, rappresentanti dei cittadini, medici legali, psicologi e assistenti sociali. L'elenco proposto dei componenti è quindi da intendere solo esemplificativo, anche se non è dichiarato come tale. 


\section{Raccomandazione 3}

La raccomandazione 3 propone la partecipazione dei genitori e di loro fiduciari ai processi decisionali dei medici e dei comitati per l'etica clinica. La partecipazione ai processi decisionali dei medici rafforza la previsione della raccomandazione 1 circa l'adozione della procedura della pianificazione condivisa, all'interno della quale il medico è chiamato a realizzare, nei confronti dei genitori e dell'eventuale fiduciario, le attività indicate nella raccomandazione 3 , che sono da attuare comunque, anche nelle situazioni, auspicabilmente rarissime, in cui non sia possibile la pianificazione condivisa. Non è facile comprendere che cosa si intenda con la partecipazione dei genitori e dei fiduciari a integrare i processi decisionali dei comitati etici. I dubbi riguardano il significato sia di "partecipazione" sia di "processi decisionali". Circa questa locuzione, rileviamo che il comitato etico non emette decisioni, intese come pronunce definitive e vincolanti, però svolge un processo in cui vaglia elementi, anche elaborati in un parere conclusivo, che équipe curante e genitori possono poi utilizzare per giungere a una loro decisione. Quanto alla partecipazione dei genitori al processo di riflessione ed elaborazione del parere del comitato etico, è opportuno che essi forniscano chiarimenti circa il loro punto di vista ma appare di più complessa attuazione un loro intervento nella discussione che porterà al parere conclusivo.

\section{Raccomandazione 4}

La raccomandazione 4 enuncia la possibilità del ricorso a una seconda opinione che può essere richiesta sia dei genitori sia dell'équipe curante. Non è specificato il significato della seconda opinione, anche se è da ritenere di natura consultiva; ovviamente, andranno comunque ricercate e valutate le ragioni di eventuali opinioni discordanti fra la seconda opinione e la prima, espressa dai curanti. Nonostante l'enfasi conferita all'"accanimento clinico", la seconda opinione non riguarda necessariamente questa tematica. E responsabilità delle équipe curante fornire l'intera documentazione clinica.

Raccomandazione 5

La raccomandazione 5 ammette, come extrema ratio, il ricorso ai giudici in caso di insanabile disaccordo fra l'équipe (questa volta medica, non più curante) e i familiari. Ciò in analogia a quanto previsto dall'art. 3, comma 4, della legge 219/2017, che però prende in considerazione solo l' $i$ potesi in cui "il rappresentante legale della persona minore rifiuti le cure proposte e il medico ritenga invece che queste siano appropriate e necessarie" [16]. La raccomandazione 5 prevede implicitamente che il ricorso al giudice debba avvenire anche nel caso in cui l'insanabile disaccordo tra équipe e genitori derivi dalla richiesta dei genitori di continuare/iniziare cure ritenute inappropriate dal medico. Nella nostra esperienza non è infrequente che i genitori non modifichino la propria posizione, nonostante l'impegno profuso per spiegare che gli interventi sul bambino da loro richiesti rappresenterebbero una ostinazione clinica, contraria ai principi dell'etica medica [17]. L'alternativa al ricorso al giudice tutelare, a nostro avviso percorribile, può essere di non somministrare trattamenti irragionevoli, anche se richiesti dai genitori, specificando dettagliatamente nella documentazione sanitaria i motivi della decisione $[18,19]$. Supporta questa posizione il disposto del comma 6 dell'art. 1 della legge 219 , per cui "Il paziente non può esigere trattamenti sanitari contrari a norme di legge, alla deontologia professionale o alle buone pratiche cliniche-assistenziali: a fronte di tali richieste il medico non ha obblighi professionali".Come il paziente non può esigere, per sé, trattamenti contrari a una buona condotta professionale, così non può esigerli il rappresentante legale del paziente minore.

Il ricorso al giudice è proponibile dopo aver esperito alcune attività, anche nuove e aggiuntive rispetto a quanto enunciato nelle prime quattro raccomandazioni. $\grave{E}$ citato un tentativo di mediazione, che non è specificato da chi debba essere gestito. I mezzi per raggiungere questa ipotetica mediazione sono tre. Il primo è "un'adeguata comunicazione" non solo con i genitori, ma anche con le persone di famiglia, o comunque "di fiducia", indicate dai genitori; l'eventuale presenza dei fratelli del bambino, anche se richiesta dai genitori del paziente, va valutata di volta in volta tenendo presente se questi abbiano raggiunto una sufficiente maturità e se il loro coinvolgimento sia opportuno visto l'impegno che comporta un processo decisionale del genere. Il secondo mezzo è il "tener conto di una corretta documentazione clinica"; è solo da osservare che qui si è voluto inserire l'aggettivo "corretta" che non figura, a proposito sempre della documentazione clinica, nella raccomandazione 4. Il terzo mezzo è costituito dalla "richiesta al comitato di etica clinica"; si fronteggiano due possibili interpretazioni, la prima è che si tratti di richiesta di mediazione rivolta al comitato, la seconda che sia da intendere in senso estensivo come considerazione di parere già espresso da parte del comitato etico, da tener presente ai fi- ni della mediazione. Aderiamo alla secon$\mathrm{da}$ interpretazione, ritenendo il ruolo del comitato etico inconciliabile rispetto all'emanazione di un parere finalizzato alla mediazione.

Circa la mediazione preliminare al ricorso giudiziario, sono ripresi, sia pur con modificazioni, spunti delle prime tre raccomandazioni, ma non è considerata la richiesta di una seconda opinione in conformità con la raccomandazione 4 .

\section{Raccomandazione 6}

La raccomandazione 6 è di dubbia opportunità nella parte in cui ipotizza una condotta medica, 1'“abbandono del bambino", in contrasto con i principi di tutela che ispirano pacificamente la professione. Anche se pleonastico, opportuno appare invece, vista la carente cultura di taluni medici in proposito, il richiamo all'accompagnamento del morire da realizzare "anche attraverso la sedazione profonda continua in associazione con la terapia del dolore".

\section{Raccomandazione 7}

La raccomandazione 7 è rivolta, in quanto richiama il principio della garanzia dell'omogeneità sul territorio, ai responsabili istituzionali dell'organizzazione dei pertinenti servizi. Tuttavia, va percepita anche dal pediatra curante come richiamo, nella concreta attività professionale, ad attivarsi personalmente per "garantire l'accesso alle cure palliative".

\section{Raccomandazione 8}

La raccomandazione 8 è pure da intendere come di portata organizzativa generale. Comunque va considerata come anche attinente all'attività pratica perché, a prescindere dalla ricerca scientifica cui la raccomandazione dedica specifica attenzione, sono comunque disponibili, e ovviamente da implementare nei singoli casi, "scale di misurazione oggettiva del dolore e della sofferenza”.

\section{Raccomandazione 9}

La raccomandazione 9 respinge il concetto che il bambino sia considerato "mero oggetto di sperimentazione e ricerca”. Essa è da intendere nel senso che esclude non che il bambino terminale possa essere oggetto di sperimentazione e ricerca, perché questa può avvenire in conformità ai princìpi dell'etica medica e alle norme di riferimento, ma che la sua sopravvivenza sia legata meramente al portare a termine un progetto di sperimentazione e ricerca.

\section{Raccomandazione 10}

La raccomandazione 10 riprende il tema della formazione dei professionisti, già ri- 
chiamata fra le funzioni del comitato per l'etica clinica. Questa raccomandazione è rivolta ai responsabili istituzionali chiamati a promuovere tale formazione e ai professionisti che dovranno attivarsi per ottenerla.

\section{Raccomandazione 11}

La raccomandazione 11 riguarda il pediatra sotto il duplice risvolto della informazione da fornire ai genitori circa la possibilità di avere agevolazioni in funzione delle condizioni del minore e la redazione di certificati adeguati.

Raccomandazione 12

La raccomandazione 12 è di carattere generale, ma può essere considerata in relazione ai singoli casi. Le associazioni citate possono essere eventualmente di supporto per l'identificazione della persona di fiducia che i genitori vorranno coinvolgere nella procedura di pianificazione condivisa delle cure oppure, più in generale, nelle attività contemplate dalla raccomandazione 3.

\section{Considerazioni conclusive}

È auspicabile la capillare diffusione delle raccomandazioni del CNB fra i professionisti sanitari, perché si tratta di strumento che potrà favorire e guidare la loro riflessione sui criteri di efficacia, appropriatezza e proporzionalità da adottare nel rispetto della dignità di bambini con limitate aspettative di vita.

Due sono i temi di peculiare rilievo operativo della mozione del CNB che meritano specifica considerazione.

Il primo è la pianificazione condivisa delle cure, valorizzata quale procedura idonea a realizzare un confronto costruttivo fra medico e genitori e la cui sistematica adozione scoraggerà i medici propensi, per "medicina difensiva", ad accogliere passivamente qualsiasi richiesta dei genitori. Il CNB non si limita così a respingere la logica della medicina difensiva, ma offre uno strumento per contrastare ipotetiche iniziative giudiziarie dei genitori, poiché la adeguata verbalizzazione, condivisa, della pianificazione delle cure può dimostrare la inconsistenza di eventuali doglianze dei genitori.

Il secondo tema di rilievo è costituito dal ruolo indefettibile dei comitati per l'etica clinica, che la mozione del CNB raccomanda siano istituiti negli ospedali pediatrici, ribadendone il ruolo esclusivamente consultivo.
Il CNB conia la locuzione "accanimento clinico" al posto di "accanimento terapeutico", un tempo adottata dal codice di deontologia medica e non soppiantata nell'uso corrente dalla espressione, utilizzata nell'art. 16 della vigente stesura di detto codice, di "interventi terapeutici clinicamente inappropriati ed eticamente non proporzionati”. Il CNB opta per una soluzione diversa; poiché i termini "accanimento" e "terapeutico" sono contraddittori [20], crea una nuova espressione, in cui è mantenuto il sostantivo "accanimento" qualificato come "clinico". Eppure, e da tempo, proprio il termine accanimento era stato respinto perché ambiguo [21] e di ardua definizione concettuale [22]. Di "accanimento clinico" il CNB dà la seguente definizione: "l'inizio di trattamenti che si presumono inefficaci o la prosecuzione di trattamenti divenuti di documentata inefficacia in relazione all'obiettivo di cura della persona malata o di miglioramento della sua qualità di vita (intesa come benessere) o tali da arrecare al paziente ulteriori sofferenze e un prolungamento precario e penoso della vita senza ulteriori benefici". Due criticità compaiono nell'incipit della definizione; infatti, è riduttivo riferirsi solo all'inizio dei trattamenti e non ai trattamenti in sé ed è troppo vaga e indeterminata l'espressione si presumono riferita alla inefficacia degli stessi. La definizione del CNB fa riferimento al concetto di inefficacia e non ai due requisiti della inappropriatezza e della non proporzionalità, valorizzati nella citata locuzione adottata nel codice di deontologia medica.

\section{$\bowtie$ anna.aprile@unipd.it}

1. Piccinni M. Prendere sul serio il problema della "capacità" del paziente dopo la 1 . n. 219/2017. Responsabilità medica 2018;3:249-68. 2. National Institute for Health and Care Excellence. End of life care for infants, children andyoung people with life-limiting conditions: planning and management. December 2016-July 2019. www.nice.org.uk/guidance/NG61.

3. Benini F, Ferrante A, Facchin P. Le cure palliative rivolte ai bambini. Quaderni acp. 2007;14: 213-7.

4. Goldman A. ABC of palliative care. Special problems of children. BMJ. 1998 Jan 3;316(7124):49-52.

5. Kanizsa S. Comportarsi in modo etico con i bambini ospedalizzati. Medic 2015;23:20-3.

6. Bellieni CV. Cure e decisioni di fine-vita in pediatria. Il Pediatra. 2019;6:56-8.

7. Giannini A, Messeri A, Aprile A, et al. End-of-life decisions in pediatric intensive care. Recommendations of the Italian Society of Neonatal and Pediatric Anesthesia and Intensive Care (SARNePI). Paediatr Anaesth. 2008 Nov;18(11):1089-95.

8. Lamiani G, Menichetti J, Fossati I, et al. Il coinvolgimento dei genitori nelle cure di fine vita: studio qualitativo in una Terapia Intensiva Pediatrica. Psicologia della Salute. 2015;3:143-54.

9. Giardina F, Gristina G. Il limite in pediatria. Responsabilità Medica. 2019;1:129-32.

10. Aprile A, Marchese V, Mazzi A, et al. Consulenza e formazione in bioetica clinica: dall'autoreferenzialità alla garanzia di qualità. Lesperienza del Laboratorio di Bioetica Clinica del Dipartimento di Medicina Molecolare dell'Università di Padova. Riv It Med Leg. 2015;1:67-85. 11. Aprile A, Piccinni M. Commento all'art. 5 (Pianificazione condivisa delle cure) della 1. 22 dicembre 2017, n. 219 (Norme in materia di consenso informato e disposizioni anticipate di trattamento). In: Gabrielli E (direttore). Commentario del codice civile. Delle persone. Vol. 2. UTET, 2019:1604-22.

12. Bolcato M, Fassina G, Sanavio M, et al. The new Italian law 219/2017: an extraordinary clinical tool in internal medicine. Italian Journal of Medicine. 2020. https://doi. org/10.4081/itjm.2020.1280

13. Benini F, Fanelli G (a cura di). Il dolore nel bambino. Strumenti pratici di valutazione e terapia. Ministero della Salute, Value Relations, 2010.

14. Benini F, Barbi E, Manfredini L. Dolore in Pediatria: miti e verità. AreaPed. 2014;15:161-72.

15. Furlan E. Comitati etici in sanità. Storia, funzioni questioni filosofiche. Franco Angeli, 2015:104-17.

16. Piccinni M. Commento all'art. 3 (Minori ed incapaci) della 1. 22 dicembre 2017, n. 219 (Norme in materia di consenso informato e disposizioni anticipate di trattamento). In: Gabrielli E (direttore). Commentario del codice civile. Delle persone. Vol. 2. UTET, 2019:1516-64.

17. Cacace $\mathrm{S}$. Il trattamento sanitario su minore o incapace: il miglior interesse del paziente vulnerabile fra (più) volontà e scienza. In Foglia M (a cura di). La relazione di cura dopo la legge 219/2017. Una prospettiva interdisciplinare. Pacini, 2019:71-80.

18. Aprile A, Bolcato M, Rodriguez D. L'assistenza nel fine vita: quali responsabilità? Un approccio medico-legale al tema. Italian Journal of Medicine. 2017;5:36-43.

19. Bolcato M, Fassina G, Rodriguez D, et al. The contribution of legal medicine in clinical risk management. BMC Health Serv Res. 2019 Feb 1;19(1):85.

20. Veronesi U. Il diritto di morire. La libertà del laico di fronte alla sofferenza. Mondadori, 2005:4.

21. Defanti CA. L'accanimento terapeutico. Janus. 2007;25:103-8.

22. Santosuosso A. Valutazione medica e autonomia del paziente: accanimento terapeutico e eutanasia. In: Barni M, Santosuosso A (a cura di). Medicina e diritto. Prospettive e responsabilità della professione medica oggi. Giuffré, 1995:188. 